Anaesthesist 2015 - 64:560-561

DOI 10.1007/s00101-015-0062-2

Online publiziert: 30 . Juli 2015

๑) Springer-Verlag Berlin Heidelberg 2015

\title{
T. Bein
}

Klinik für Anästhesiologie, Klinikum der Universität Regensburg, Regensburg, Deutschland

\section{"Move to improve"}

\section{Neue Leitlinie zu Lagerungstherapie und Frühmobilisation}

ne der Verhinderung eines generellen „deconditioning" (Schwäche, Ermüdbarkeit, Atrophie der Atem- und Skelettmuskeln, psychokognitive Defizite und Delir, [2]) als (überlebens)-vorteilhaft, wenn diese Maßnahmen in ein allgemeines Bündel von reduzierter und angepasster Sedierung sowie Förderung der frühen Spontanatmung eingebunden wurden [1].

\section{Paradigmenwechsel} wissenschaftliche (Erforschung des Wirkmechanismus) und klinisch-wissenschaftliche Anstrengungen (Nachweis eines Überlebensvorteils) zur Untersuchung der Beatmung in Bauchlage bei Patienten mit akutem Lungenversagen statt - mit wechselhaftem Erfolg: Während die physiologischen Mechanismen der Bauchlagerung mithilfe bildgebender und nuklearmedizinischer Techniken gut erfasst wurden, dauerte es lange, bis eine eindeutige klinische Einordnung (Indikationen, Durchführung, Outcome) möglich war.

Die Deutsche Gesellschaft für Anästhesiologie und Intensivmedizin e. V. (DGAI) beauftragte 2005 eine Leitliniengruppe, die bis dato vorliegenden Erkenntnisse der Bauchlagerung, der kontinuierlichen lateralen Rotationstherapie, der Seiten- und der Oberkörperhochlagerung in einer S2e-Leitlinie zusammenzufassen. Die Revision war nun 2014 fällig, und die Leitliniengruppe nahm diese Überprüfung zum Anlass, den Aspekt der Frühmobilisation von Patienten aufzunehmen. Diese Erweiterung bot sich an. In den letzten Jahren war zunehmend die frühe und schrittweise Mobilisation von kritisch kranken Patienten propagiert und durch gute wissenschaftliche Studien untermauert worden [z. B. 5]: Die Förderung der Bewegungsfähigkeit innerhalb von $72 \mathrm{~h}$ nach Aufnahme erwies sich im Sin-
Die aktuelle Aufnahme des Aspekts der Frühmobilisation war der Leitliniengruppe ein besonderes Anliegen: Hier bahnt sich ein Paradigmenwechsel an, der vom immobilisierten, (zu) tief sedierten Intensivpatienten wegführt zum wachen Patienten an der Bettkante oder neben dem Bett stehend - möglicherweise auch gehend mit Beatmung. Die Leitlinie gibt praktische Hinweise für die Ein- und Ausschlusskriterien sowie für Sicherheitsaspekte dieser anspruchsvollen Maßnahme. Besonderes Augenmerk wird auf technische und personelle organisatorische Voraussetzungen gelegt - auch im Sinne interdisziplinärer Zusammenarbeit mit Physiotherapeuten: Sicherheit und Wohl der Patienten müssen oberste Maxime sein, damit sich der Überlebensvorteil durch Frühmobilisation entfalten kann.

Auch die Bauchlagerung erfährt in dieser Revision eine besondere klinische Würdigung, da durch aktuelle hochwertige prospektive randomisierte Studien (z. B. [3]) und Metaanalysen gezeigt wurde, dass die frühzeitige ( $<48 \mathrm{~h}$ nach Erkrankungsbeginn) und ausreichend dauernde ( $\geq 16 \mathrm{~h} / \mathrm{Tag}$ ) Bauchlagerung die Überlebensprognose von Patienten mit „acute respiratory distress syndrome“ (ARDS) erheblich verbessern kann. Die
Oberkörperhochlagerung hingegen musste diesmal eine gewisse „Rückstufung“ hinnehmen. Die noch in der ersten Version hochgradig empfohlene $45^{\circ}$-Oberkörperhochlagerung für alle beatmeten Patienten wurde abgemildert zu einer $20^{\circ}$ - bis $45^{\circ}$-Oberkörperhochlagerung unter Berücksichtigung von Einschränkungen und Kontraindikationen. Es hatte sich abgezeichnet, dass dieses rigide Konzept im klinischen Alltag nicht durchführbar war. Darüber hinaus zeigten weitere Studien, dass unerwünschte Wirkungen (Hypotension, Selbstgefährdung des Patienten, Hautläsionen) wohl zuvor unterschätzt worden waren.

\section{Was ist neu an dieser Leitlinie?}

Die erstmalige Beschreibung und klinisch-wissenschaftliche Einordnung der Frühmobilisation soll einen generellen Wandel in der Behandlung von kritisch kranken Patienten einleiten: von prolongierter Immobilisierung zu „move to improve"! Die Bauchlagerung erfährt eine weitere Stärkung und stellt eine hochgradig empfehlenswerte Maßnahme zur Outcome-Verbesserung bei ARDS dar. Die Oberkörperhochlagerung verlässt die rigide "Anordnung“ einer halbsitzenden $45^{\circ}$-Position und erfährt individuelle Anpassungen. Die Leitliniengruppe ist gespannt, wie sich diese Themen in den nächsten Jahren entwickeln werden.

Der Beitrag in der Rubrik „Leitlinien und Empfehlungen" dieser Ausgabe von Der Anaesthesist fasst die Kernaussagen der neuen Leitlinie zusammen.

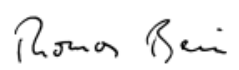

T. Bein 


\section{Korrespondenzadresse}

Prof. Dr. T. Bein

Klinik für Anästhesiologie

Klinikum der Universität Regensburg

93042 Regensburg

thomas.bein@ukr.de

\section{Einhaltung ethischer Richtlinien}

Interessenkonflikt. T. Bein gibt an, dass kein Interessenkonflikt besteht.

\section{Literatur}

1. Adler J, Malone D (2012) Early mobilization in the intensive care unit: a systematic review. Cardiopulm Phys Ther J 23:5-13

2. Brower RG (2009) Consequences of bed rest. Crit Care Med 37:S422-S428

3. Guerin C, Reignier J, Richard J, Beuret P, Gacouin A, Boulain T et al (2013) Prone positioning in severe acute respiratory distress syndrome. N Engl J Med 368:2159-2168

4. Piehl MA, Brown RS (1976) Use of extreme position changes in acute respiratory failure. Crit Care Med 4:13-14

5. Schweickert WD, Pohlman MC, Pohlman AS, Nigos C, Pawlik A, Esbrook CL et al (2009) Early physical and occupational therapy in mechanically ventilated, critically ill patients: a randomised controlled trial. Lancet 373:1874-1882

\section{Die Notfallversorgung muss optimiert werden}

\section{Klausurtagung der DIVI}

Mehr als 20 Millionen Menschen kommen

in Deutschland jedes Jahr in die Notaufnahmen und Notfallambulanzen deutscher Krankenhäuser, viele Kliniken versorgen schon jetzt mehr als 100 Notfallpatienten pro Tag - Tendenz steigend. Die Kosten für die Behandlung werden den Krankenhäusern nur teilweise vergütet, da das für Krankenhauspatienten eingeführte DRG-Abrechnungssystem aufgrund seiner Struktur planbare Patienten begünstigt, während die Versorgung von Notfallpatienten hohe Vorhaltekosten erfordert. Darunter leiden besonders Krankenhäuser, die einen hohen Anteil an Patienten mit Akuterkrankungen aber auch mit chronischen Grunderkrankungen behandeln. Deshalb ging es auf der diesjährigen Klausurtagung der Deutschen Interdisziplinären Vereinigung für Intensiv- und Notfallmedizin (DIVI) unter der Leitung des aktuellen Präsidenten Prof. Gerhard Jorch am 19. Juni in Hannover um das Thema „Perspektiven und Visionen der Notfallmedizin".

Jeder sechste Patient, der in eine Notaufnahme kommt, ist lebensbedrohlich erkrankt oder verletzt. Da die Bevölkerung in Deutschland immer älter und dadurch kränker wird, ist zukünftig mit einem noch größeren Patientenaufkommen in den Notaufnahmen zu rechnen. „Deshalb ist es unser Ziel, eine flächendeckende Verbesserung und nachhaltige Finanzierung der Versorgung von Patienten mit akuten Erkrankungen und Verletzungen zu erreichen", sagt Privat-Dozent Dr. Hans-Jörg Busch. Interdisziplinäre Notaufnahmen, idealerweise in enger Zusammenarbeit mit den kassenärztlichen Notdiensten und dem Notarzt- bzw. Rettungsdienst, sind dafür am besten geeignet. Es müssen dabei Modelle entwickelt werden, die dem jeweiligen Leistungsvolumen der Krankenhäuser gerecht werden.

„Eine optimale Patientenversorgung erfordert die fachärztliche Kompetenz aus den Fächern Anästhesie, Innere Medizin, Chirurgie, Neuromedizin und Kinderheilkunde mit spezieller notfallmedizinischer Schulung wie sie bereits jetzt durch das DIVI-Curriculum
Notfallmedizin erfolgt und perspektivisch mit einer Zusatzweiterbildung Notfallmedizin durch die Bundesärztekammer geplant ist", erklärt Prof. Andreas Seekamp, Sprecher der DIVI- Sektion „Interdisziplinäre Notaufnahme“.,Deshalb wäre es überaus sinnvoll, wenn Ärzte unterschiedlicher Fachrichtungen bereits während ihrer Weiterbildung verpflichtend eine angemessene Zeit in einer zentralen Notaufnahme arbeiten würden. Hinzu kommen infrastrukturelle Notwendigkeiten, wie die Beseitigung baulicher Defizite und auch eine bessere Kommunikation aller Beteiligten." Denn nur so lassen sich aus Expertensicht die Aufgaben der Notfallmedizin optimal umsetzen. „Eine große Hilfe können gerade bei zeitkritischen Notfällen interdisziplinär und interprofessionell mit den wesentlichen an der notfallmedizinischen Versorgung Beteiligten abgestimmte Algorithmen sein", erklärt Professor André Gries. „Es handelt sich um Checklisten, die die notwendigen Diagnostik- und Behandlungsabschnitte, die optimalen medizinischen Maßnahmen, aber auch organisatorische Punkte in zeitlicher Folge vorgeben und gerade auch bei einem hohen Aufkommen von Akutpatienten ein wertvolles Werkzeug sein können. Sie können gerade jüngeren Kollegen dabei helfen, auch in einer unübersichtlichen Situation strukturiert zu handeln. Dennoch: Im Vordergrund steht eine gute Qualifikation, weshalb die DIVI auch eine Zusatzweiterbildung für Notaufnahmen bei der Bundesärztekammer beantragt hat."

Fazit

Angesichts der stetig steigenden Zahl an Notfällen, vor allem bei älteren Menschen und der gleichzeitig sinkenden Bettenzahl, steht die Notfallmedizin vor großen Herausforderungen - sowohl medizinisch als auch finanziell. Es besteht Handlungsbedarf.

DIVI, Berlin, 25. Juni 2015 